Erschienen in: Behr, Janina/Conrad, François/Kornmesser, Stephan/Tschernig, Kristin (Hrsg.): Schnittstellen der Germanistik. Festschrift für Hans Bickes.

Berlin/Bern/New York: Lang, 2020, S. 185-214.

DOI: https://doi.org/10.3726/b16845

\title{
POSTPRINT
}

\section{Christine Möhrs}

\section{„Hast du eine Ahnung, ...?" Eine lexikografische und korpusbasierte Untersuchung am Beispiel des Lexems Ahnung}

\begin{abstract}
The article focuses on the lexeme Ahnung. A lexicographic analysis shows the range of information offered by Ahnung in selected dictionaries, especially monolingual DaF dictionaries, and displays how the spectrum of meaning is represented in them. Corpusbased analyses from two samples from FOLK and DEREKo investigate exemplary form characteristics and, with regard to the written-language data, the occurrence in text types. Ahnung shows a slightly higher combinatorial potential in written-linguistic data than in spoken-linguistic data. A clear tendency to the connection keine Ahnung is however to be recognized in both data sets.
\end{abstract}

\section{Einleitung}

"Hast du eine Ahnung, worum es in diesem Beitrag geht?" - „Keine Ahnung!" Auf diese Frage soll in der Einleitung eine Antwort gefunden und erläutert werden, welche Hintergründe für diesen Artikel relevant sind, welcher Fragestellung nachgegangen wird, welchen Beitrag der Artikel aus der Perspektive Lexikografie und Korpuslinguistik leisten möchte und wie er aufgebaut ist.

Die Bedeutung eines Wortes und vor allem auch eines abstrakten Wortes zu entschlüsseln, stellt für SprecherInnen im Allgemeinen, aber insbesondere für LernerInnen des Deutschen als Zweit- bzw. Fremdsprache eine Herausforderung dar. Dies wurde in verschiedenen Studien nachgewiesen (vgl. z.B. Meliss 2015: 418f. oder Meliss et al. 2019: 137). In diesen Studien wird deutlich, dass die Bedeutungsentschlüsselung und die Frage danach, wie ein Wort in einen Kontext eingebettet ist (wie also sein syntagmatisches Potenzial ausgestaltet ist), unter anderem sehr häufige Gründe sind, warum Nachschlagende ein Wörterbuch konsultieren. Die beiden genannten Aspekte spielen im Zusammenhang des Spracherwerbs auch eine wichtige Rolle: Die Forschung zeigt, dass beim Erlernen einer Sprache zwar auch einzelne Einheiten als solche erfasst und gelernt werden, dass aber gerade der Kontext und die Bildung von komplexeren Einheiten einen entscheidenden Anteil im Spracherwerb haben. So stellt z.B. Feilke (2009) fest: 
„Wörter und Wendungen stehen in Handlungszusammenhängen und Textzusammenhängen, und sie werden rezeptiv wie produktiv aus diesen heraus erworben. In der Abfolge von Kennen, Lernen und Können wird der Handlungskontext eines Ausdrucks zunehmend durch den Erwerb seiner innersprachlichen und satzbezogenen Kontexte erweitert." (Feilke 2009: 4)

Der vorliegende Artikel greift das abstrakte Lexem Ahnung auf und widmet sich seinem Vorkommen, wie es in den Bsp. (1)-(4) in authentischer Interaktion oder in authentischen geschriebenen Texten auszumachen ist. ${ }^{1}$

(1) FOLK_E_00084_SE_01_T_02_DF_012

0726 EG [((gähnt))]

$0727 \quad(0.4)$

0728 FR ja da musst schon gähnen ne wie ich hör[e ((lacht))]

0729 EG [ja äh keine ahnung ich] bi ich weiß auch gar nich wieso (.) ich hab gar nich so wenig geschlafen (.) ging doch eigentlich

0730 FR ${ }^{\circ} \mathrm{h}(($ lacht $))$

$0731 \quad(0.42)$

0732 EG ah wieso war dieser zahnarzttermin auch so früh (.) vollkommen sinnlos

(2) FOLK_E_00055_SE_01_T_07_DF_01

$0355 \mathrm{NH}$ un dann ich glaub die waren einfach die waren

$0356 \quad(0.31)$

$0357 \mathrm{NH}$ verärgert dass ich zu nem andern anbieter gewechselt hatte oder so die ham das nich so gern gesehen jedenfalls meinte die ja aso ${ }^{\circ} \mathrm{hh}$ [waren sie] bei diesen deppen bei o two ${ }^{\circ} \mathrm{h}$ [die ham ja] keine ahnung ${ }^{\circ} \mathrm{h}$ und überhaupt da (.) de[s muss] ganz anders laufen sie müssen erst hier kündigen und dann können sie ers[t_n neuen ver] trag machen [und überhaupt ${ }^{\circ} \mathrm{h}$ ] ich also mit dieser information wieder zurück [zu o two $\mathrm{zu}$ dem gleichen] bearbeiter ${ }^{\circ} \mathrm{h}$ a die ham ja keine ahnung wir machen des immer so ne des ge funktioniert immer also ${ }^{\circ} \mathrm{h}$ (.) könn_se den ruhig sagen dass das ne ganz normale proze[dur is un die sollen sich nich so anstellen]

1 Die Hervorhebung von ahnung/Ahnung in den Korpusbeispielen stammt von C. Möhrs und dient dem besseren Auffinden des Lexems in den Beispielen.

2 Ein kurzer Hinweis zu den Transkripten aus dem „Forschungs- und Lehrkorpus Gesprochenes Deutsch" (= FOLK), in diesem Beitrag: Da prosodische Eigenarten in dieser Analyse nicht betrachtet werden, wird auf das Minimaltranskript aus der "Datenbank für Gesprochenes Deutsch“ (= DGD) zurückgegriffen. Elemente in doppelten runden Klammern, z.B. ((gähnt)) = nonverbale Kommunikationselemente; Zahlen in runden einfachen Klammern, z.B. (0.42) = Pausen im Gespräch; Punkt in einfacher runder Klammer, (.) = Mikropause; Elemente in eckigen Klammern, z.B. im Bsp. (1) in Zeilen $728([$ e ((lacht) $)])$ und 729 ([ja äh keine ahnung ich $])=$ Äußerungsteile laufen parallel ab; Atmen $=\mathrm{z} . \mathrm{B} .{ }^{\circ} \mathrm{h} /{ }^{\circ} \mathrm{hh}$. 
(3) DEREKo: Süddeutsche Zeitung, 23.10.2004, S. V3/9

Als Jonas im Frühsommer bei Ad Mission begann, hatte er von Photoshop und Pixeln keine Ahnung. Jetzt, ein halbes Jahr später, ist er ein gefragter Logoentwickler: Zuerst hat er das Layout für ein neues Computerspiel entwickelt. Dann durfte er sich an einem Firmenlogo für einen Knochenzementhersteller wagen.

(4) DEREKo: die tageszeitung, 09.07.2007, S. 14; Klimaschutz per SMS

Das ist das Mitreißende an Al Gores großer Klimaschutzkampagne: Anders als bei ihren Vorbildern, dem "Live Aid" beziehungsweise dem "Live8"-Konzert, ist eine Vereinfachung der Probleme hier weniger unzulässig. Der weltweite Hunger davon hat die Öffentlichkeit mittlerweile eine Ahnung - kann mit Hilfslieferungen nicht bekämpft werden. Oft bewirken sie sogar genau das Gegenteil, etwa wenn Kleiderspenden lokale Märkte zerstören.

Einerseits soll der Dokumentation von Bedeutung/Funktion und Gebrauch zu Ahnung ${ }^{3}$ in Wörterbüchern nachgegangen werden, um zu sehen, was Nachschlagende zu diesem Lexem darin an Informationen finden können (vgl. Abschnitt 3.1). Auf Basis von Korpusdaten soll andererseits der Gebrauch des Lexems Ahnung untersucht werden, um etwas über Ahnung in authentischen Texten und Gesprächen (Interaktionen) zu erfahren.

Für die Analyse von authentisch gesprochen-sprachlichem Material wurde das „Forschungs- und Lehrkorpus Gesprochenes Deutsch" (=FOLK $\left.{ }^{4}\right)$ ausgewählt (vgl. Abschnitt 3.2), für authentisch geschriebenes Material das „Deutsche Referenzkorpus“ (= DEREKo $\left.{ }^{5}\right)$ (vgl. Abschnitt 3.3) ${ }^{6}$. Beide Korpora sind am Leibniz-Institut für Deutsche Sprache (= IDS) erstellt worden und werden über Korpusanalysetools (COSMAS $\mathrm{II}^{7}, \mathrm{DGD}^{8}$ ) verfügbar gemacht. Die Erkenntnisse aus der Analyse von Wörterbüchern sowie aus der Analyse von authentischem Datenmaterial werden in Abschnitt 4 in Kombination betrachtet.

3 Ahnung ist Teil des Wortschatzes des Goethe-Zertifikats Bl (siehe Duden-online: URL: https://www.duden.de/rechtschreibung/Ahnung [letzter Zugriff: 02.08.2019]).

4 FOLK (Forschungs- und Lehrkorpus Gesprochenes Deutsch): URL: http://agd. ids-mannheim.de/folk.shtml [letzter Zugriff: 02.08.2019].

5 DeReKo (Deutsches Referenzkorpus): URL: http://wwwl.ids-mannheim.de/kl/ projekte/korpora.html [letzter Zugriff: 02.08.2019].

6 Für die Mithilfe bei der Datenanalyse und -kodierung danke ich sehr herzlich Mojenn Schubert (stud. Hilfskraft im LeGeDe-Projekt).

7 COSMAS II (Corpus Search, Management and Analysis System): URL: https://www.ids-mannheim.de/cosmas2/, [letzter Zugriff: 02.08.2019].

8 DGD (Datenbank für Gesprochenes Deutsch): URL: https://dgd.ids-mannheim.de/, [letzter Zugriff: 02.08.2019]. 
Die Betrachtung von authentisch gesprochen-sprachlichem Material und das Herausarbeiten von lexikalischen Bedeutungen sowie Funktionen von Verwendungsweisen in der Interaktion entspringt dem Forschungsprojekt "Lexik des gesprochenen Deutsch" ( $=$ LeGeDe), einem Drittmittelprojekt am IDS. ${ }^{9}$ Ziel dieses Projektes ist die Erstellung eines Prototyps einer lexikografischen Ressource, in der auf Basis von Daten aus FOLK lexikalische Besonderheiten ausgewählter Lemmata im gesprochenen Deutsch herausgearbeitet werden sollen. Geleitet von einem mixed-method-Ansatz werden neben lexikologischen Analysen zur Herausarbeitung des Bedeutungsspektrums interaktionslinguistische Sequenzanalysen durchgeführt. Mit der Erstellung einer lexikografischen Ressource auf Basis von gesprochen-sprachlichem Material betritt das Projekt Neuland, da es eine solche Art von Ressource weder für das Deutsche noch für eine andere Sprache gibt. Die zur Verfügung stehende Datenbasis (FOLK) ist in den letzten Jahren so ausgebaut worden, dass sie eine gute und auch hinreichend große Ausgangsbasis für die Anlage einer korpusbasierten Wörterbuchressource bietet. Die gesprochen-sprachlichen Daten werden in der Abteilung Pragmatik am IDS gepflegt und ausgebaut. Das LeGeDe-Projekt reiht sich mit dem lexikografischen Vorhaben in eine schon länger am IDS in der Abteilung Lexik bestehende Tradition ein, Internetwörterbücher zu entwickeln. Über das Portal OWID ${ }^{10}$, dem Online Wortschatz Informationssystem Deutsch, sind in den letzten Jahren zahlreiche Wörterbuchressourcen unterschiedlichster Art zugänglich gemacht worden. Die LeGeDe-Ressource, als eine Art Prototyp, ist über die Plattform OWID ${ }^{\text {plus1! }}$ abrufbar, auf der Ressourcen und Daten mit stärker experimentellem Charakter zugänglich gemacht werden.

In Forschungsarbeiten zum gesprochenen Deutsch gibt es zu Ahnung bzw. zu der laut Wörterbüchern gebräuchlichen Verbindung keine Ahnung noch wenige ausführliche und systematische Beschreibungen (vgl. z.B. König/Stoltenburg

9 Es handelt sich um ein von der Leibniz-(Gemeinschaft drittmittelgefördertes Projekt mit einer Laufzeit von drei Jahren (2016 bis 2019). Das Projekt wird als Kooperationsprojekt der Abteilungen Lexik und Pragmatik durchgeführt (Leitung: Prof. Dr. Meike Meliss [IDS/USC, Universidad de Santiago de Compostela], Dr. Christine Möhrs [IDS]) und es arbeiten im Team LexikografInnen, Interaktionslinguistinnen, ComputerlinguistInnen sowie KorpuslinguistInnen. Näheres zum Projekt: vgl. Meliss/Möhrs (2017), Möhrs et al. (2017) oder auf der Projektwebseite: URL: http:/www.ids-mannheim.de/ lexik/lexik-des-gesprochenen-deutsch.html, [letzter Zugriff: 02.08.2019].

10 OWID (Online-Wortschatz-Informationssystem Deutsch): URL: https://www.owid.de/, [letzter Zugriff: 02.08.2019].

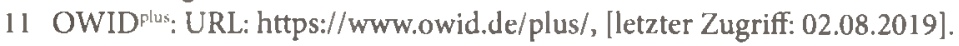


2013: 11, Bergmann 2017: 160, Eichinger 2018: 258ff.). In Ergänzung zu dem oben genannten Analyseansatz im LeGeDe-Projekt wurde für diesen Beitrag noch schriftsprachliches Material hinzugezogen. Ergebnisse aus den gesprochenen und den geschriebenen Daten werden an ausgewählten Stellen miteinander verglichen.

\section{Methodisches Vorgehen}

Für die Wörterbuchanalyse wurden vier einsprachige deutsche Wörterbücher ausgewählt (vgl. Abschnitt 3.1) und darin wurde der jeweilige Wörterbuchartikel zum Lemma Ahnung betrachtet. Für die Analyse von gesprochen-sprachlichen Daten wurde eine Zufallsstichprobe $(\mathrm{n}=300)$ aus FOLK zu Ahnung aus der Gesamttreffermenge (Stand 05.02.2019: 576 Treffer gesamt, DGD-Release 2.11) untersucht, für die Analyse von geschrieben-sprachlichen Daten wurde ebenfalls eine Zufallsstichprobe ( $\mathrm{n}=300$ ) zum Lemma Ahnung gezogen (Stand 27.02.2019: 181.541 Treffer gesamt, DeReKo-2018-II). An ausgewählten Stellen werden zur Untersuchung des Kombinationspotenzials Bigram-Analysen (also die direkte Abfolge von zwei sprachlichen Elementen) aus beiden Korpora über die gesamte Datenmenge hinzugezogen. Diese Datenlage ermöglicht es, exemplarische Analysen zu Vorkommens- und Formbesonderheiten von Ahnung sowohl auf Basis von gesprochenem als auch von geschriebenem Material durchzuführen.

\section{Analyse des Lexems Ahnung}

\subsection{Ahnung in Wörterbüchern}

Welches Bild zeigt sich zum Bedeutungsspektrum von Ahnung in einsprachigen Wörterbüchern zum Deutschen? In Tab. 1 ist eine Übersicht aus vier verschiedenen Wörterbüchern dargestellt. Das Langenscheidt-Großwörterbuch Deutsch als Fremdsprache (= LGW-DaF), PONS Online Wörterbuch DaF (= PONS-DaF) sowie Duden-Deutsch als Fremdsprache (= Duden-DaF) repräsentieren dabei LernerInnenwörterbücher. Hinzugenommen ist in der Übersichtsdarstellung außerdem noch das Duden-Universalwörterbuch/Duden-online (= Duden-online), das als allgemeines Bedeutungswörterbuch eingestuft werden kann und auch von vielen L2-SprecherInnen häufig verwendet wird (vgl. z.B. Taborek 2019: 214 oder Meliss et al. 2019: 107). 
Tab. 1: Ubersicht zu Ahnung in Wörterbüchern (LGW-DaF, PONS-DaF, Duden-DaF, Duden-online)

\begin{tabular}{|c|c|}
\hline $\begin{array}{l}\text { Worter- } \\
\text { buch }\end{array}$ & Information zu Ahnung \\
\hline \multirow[t]{4}{*}{$\begin{array}{l}\text { LGW- } \\
\text { DaF }\end{array}$} & $\begin{array}{l}\text { ein vages Gefühl von einem (bevorstehenden) Ereignis oder von einer Sache, } \\
\text { über die man nichts Genaues weiß } \\
\text { - <eine böse, dunkle, düstere Ahnung; eine Ahnung befällt, überkommt } \\
\text { jemanden> } \\
\text { - Meine Ahnungen haben nicht getrogen waren richtig } \\
\text { - Ich hatte schon so eine Ahnung, dass das nicht klappen wird }\end{array}$ \\
\hline & $\begin{array}{l}\text { (von etwas) eine Ahnung haben } \\
\text { etwas wissen (weil man es mitgeteilt bekam oder selbst erlebt hat) oder sich } \\
\text { etwas vorstellen können } \\
\text { - <von etwas nicht die entfernteste, geringste, leiseste, mindeste } \\
\text { Ahnung haben> } \\
\text { - Hast du eine Ahnung, wie er heißt? } \\
\text { - Ich hatte ja keine Ahnung, dass du schon zurück bist } \\
\text { - Habt ihr eine Ahnung, wie der Unfall passiert ist? } \\
\text { [Info: meist in verneinter oder fragender Form] }\end{array}$ \\
\hline & $\begin{array}{l}\text { (von etwas) eine Ahnung haben } \\
\text { in einem Bereich Kenntnisse haben, die man durch Lernen erworben hat } \\
\text { - <von etwas keine, wenig, viel Ahnung haben> } \\
\text { - Er hat von Technik absolut keine Ahnung }\end{array}$ \\
\hline & $\begin{array}{l}\text { Hast 'du eine Ahnung! gesprochen Da täuschst du dich aber!; Keine Ahnung! } \\
\text { gesprochen verwendet als Antwort, um zu sagen, dass man etwas nicht weiß }\end{array}$ \\
\hline \multirow[t]{2}{*}{$\begin{array}{l}\text { PONS- } \\
\text { DaF }\end{array}$} & $\begin{array}{l}\text { Vorgefühl, der Sachverhalt, dass man etwas Zukünftiges bereits spürt, ohne } \\
\text { Genaues darüber zu wissen } \\
\text { - eine bestimmte / böse / ungewisse Ahnung haben } \\
\text { - Erwartung einer unangenehmen Sache; schlimme Ahnung }\end{array}$ \\
\hline & $\begin{array}{l}\text { (kein Plur., umg. }{ }^{12} \text { ) } \\
\text { eine gewisse Vorstellung oder Kenntnis } \\
\text { - von etwas viel/(überhaupt) keine Ahnung haben } \\
\text { - Weißt du, wo mein Schlüssel ist? Keine Ahnung! } \\
\text { - null Ahnung umg (überhaupt keine Ahnung) } \\
\text { - Dieser Ignorant hat doch keine Ahnung! } \\
\text { - nicht den Hauch einer Ahnung haben } \\
\text { - Von Physik hatte er wirklich null Ahnung! } \\
\text { - von Tuten und Blasen keine Ahnung haben umg (überhaupt keine } \\
\text { Ahnung haben) }\end{array}$ \\
\hline
\end{tabular}

12 „umg.“ = umgangssprachlich 
Tab. 1: Fortsetzung

\begin{tabular}{|c|c|}
\hline & Information zu Ahnung \\
\hline & $\begin{array}{l}\text { - jemand hat (von etwas) nicht die leiseste Ahnung (jd weiß gar nichts } \\
\text { (von etwas)) } \\
\text { - Ich habe nicht die mindeste Ahnung von dieser Thematik. } \\
\text { - Eigentlich hat er keine Ahnung, er redet ihr alles nur nach. }\end{array}$ \\
\hline $\begin{array}{l}\text { Duden- } \\
\text { DaF }\end{array}$ & $\begin{array}{l}\text { gefühlsmäßige Erwartung (in Bezug auf etwas, was geschehen, } \\
\text { eintreten wird) } \\
\text { - eine Ahnung des kommenden Unheils; } \\
\text { - meine Ahnung hat mich nicht getrogen } \\
\text { - keine Ahnung haben (ugs.): nicht wissen: ich habe keine Ahnung, wo } \\
\text { sie ist. }\end{array}$ \\
\hline $\begin{array}{l}\text { Duden- } \\
\text { online }\end{array}$ & $\begin{array}{l}\text { undeutliches, dunkles Vorgefühl } \\
\text { - eine dunkle, düstere Ahnung } \\
\text { - eine Ahnung des kommenden Unheils } \\
\text { - meine bösen Ahnungen trogen mich nicht }\end{array}$ \\
\hline & $\begin{array}{l}\text { intuitives Wissen, Vermutung, Vorstellung von etwas } \\
\text { - von etwas absolut keine Ahnung, keine blasse, nicht die geringste, } \\
\text { mindeste, entfernteste Ahnung haben } \\
\text { - hast du eine Ahnung (umgangssprachlich; weißt du), wo sie } \\
\text { hingegangen ist? } \\
\text { - keine Ahnung (umgangssprachlich; als Antwort auf eine Frage; ich } \\
\text { weiß es nicht) } \\
\text { - hast du eine Ahnung! (umgangssprachlich; da irrst du dich aber sehr!; } \\
\text { wenn du wüsstest!) } \\
\text { - eine Ahnung von etwas bekommen } \\
\text { - der Bericht vermittelt eine Ahnung davon, was sich damals abgespielt } \\
\text { hat }\end{array}$ \\
\hline
\end{tabular}

Auf der Basis dieser Informationen aus Wörterbüchern lässt sich für das Lexem Ahnung folgende Bedeutungsmatrix herausarbeiten (vgl. Abb. 1), von der nicht immer alle Bedeutungen in den Wörterbüchern aus Tab. 1 gleichermaßen abgebildet werden. Das LGW-DaF differenziert in den Bedeutungen am feinsten, das Wörterbuch Duden-DaF am gröbsten. 


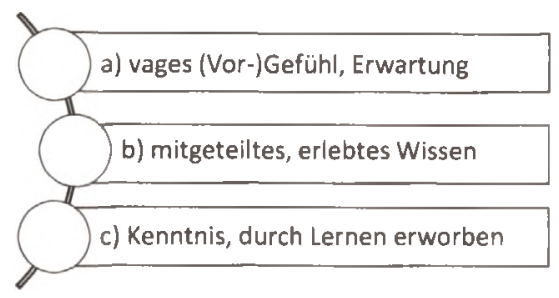

Abb. 1: Bedeutungsspektrum von Ahnung (Basis: ausgewählte Wörterbücher, vgl. Tab. 1)

Die Bedeutungen b) und c) lassen sich nicht an allen Stellen wirklich gut voneinander unterscheiden. PONS-DaF, als Spezialwörterbuch für L2-SprecherInnen, und Duden-online, als allgemeines Bedeutungswörterbuch, trennen in der Bedeutungsdisambiguierung bei diesen beiden Lesarten auch nicht. Duden-DaF setzt nur die Bedeutung a) an, was etwas verwunderlich ist, da die Lesarten b) und c) in den Daten recht dominant erscheinen (vgl. hierzu Abschnitt 4). Das LGW-DaF ist von den untersuchten Wörterbüchern das einzige, in dem in der Bedeutungsdisambiguierung zwischen a) und b) differenziert wird.

\subsection{Ahnung in FOLK}

FOLK ist das größte Korpus für gesprochenes Deutsch in der Interaktion (vgl. Schmidt 2014a, b). FOLK ist integriert in das „Archiv für Gesprochenes Deutsch“ (AGD) und kann für Recherchen über die Datenbank für Gesprochenes Deutsch (DGD) abgerufen werden. Nach dem aktuellen Release umfasst FOLK in der DGD Version 2.12 (Stand: Mai 2019) (vgl. Schmidt 2017) rund 2,4 Mio. Tokens bei ca. 250 Std. Audioaufnahmen mit teils auch Videomaterial. Das Korpus ist zusammengesetzt aus Gesprächsaufnahmen und entsprechenden Transkripten im deutschsprachigen Raum in unterschiedlichen privaten, institutionellen und öffentlichen Kontexten. Die Daten zeichnen aus, dass sie medial mündlich einzustufen sind und durch die verschiedenen enthaltenen Gesprächskontexte ein Spektrum eines Nähe-Distanz-Kontinuums von sprachlich-kommunikativen Handlungen abdecken (vgl. dazu Koch/Oesterreicher 2019: 194). Sie repräsentieren authentische (nicht elizitierte), spontane (nicht vorbereitete) und standardnahe Sprache. Die Aufnahmen stammen aus den Jahren 2003 bis 2016, wobei der Großteil der Daten aus Aufnahmen nach 2010 besteht. Die Transkripte können nach unterschiedlichen Annotationsebenen durchsucht werden, außerdem ist es NutzerInnen der DGD möglich, sich zu den Ergebnissen einer 
strukturierten Tokensuche mit Konkordanzen Metadaten zu SprecherInnen und Gesprächsereignissen anzeigen zu lassen. Darüber hinaus können Suchen auch über Metadatenparameter gezielt eingeschränkt werden.

Für die vorliegende Untersuchung wurde eine Zufallsstichprobe aus FOLK gezogen (im DGD Release 2.11, Stand: 30.11.2018) die insgesamt 300 Treffer umfasst. Dabei wurden keine Sprechereignisse im Vorfeld ausgeschlossen, um ein unvoreingenommenes Bild der Vorkommen von Ahnung im Korpus zu gewinnen. Von den 300 Treffern der Stichprobe konnten 275 Treffer als analysierbar eingestuft und damit weiter ausgewertet werden. Die restlichen Treffer ( $\mathrm{n}=25)$ sind vor allem aus Gründen der Verständlichkeit aus der Menge der analysierbaren Fälle herausgefallen, weil z.B. Ahnung oder der direkte Kontext von Ahnung verrauscht ist oder Gesprächsbeiträge überlappt sind. ${ }^{13}$

Zur Analyse der Daten wird auf zwei Aspekte genauer eingegangen: (i) das kombinatorische Potenzial von Ahnung (insbesondere bezogen auf das direkt links vorkommende Element in Form von Artikeln, Adjektiven, Zahladverbien etc.), (ii) die Betrachtung der Kombination keine Ahnung als eine zentrale Verbindung unter verschiedenen Gesichtspunkten (syntagmatische Einbettung, Formbesonderheiten, inhaltliche Orientierung an den umliegenden und damit einhergehend die Stellung im Äußerungskontext).

(i) Für die Betrachtung des Elementes direkt vor dem Lemma Ahnung, welches in der für diesen Beitrag gezogenen Stichprobe ausschließlich im Singular vorkommt, wurden die 275 gültigen Treffer ausgewertet (vgl. Abb. 2). Es zeigt sich mit 96,7\% eine sehr klare Tendenz zu dem Determinativum kein- (hierunter sind alle Transkriptionsvarianten wie keine, keene, kei, ko gefasst). Auf den indefiniten Artikel ein-mit 1,8\% folgen die unbestimmten Zahlwörter viel und wenig sowie das Adjektiv richtig- mit je $0,4 \%$ Vorkommen.

13 Beispielsweise wird zum Anonymisieren über Eigenname ein Rauschen gelegt, was teils dazu führt, dass ein Kontext nicht mehr gut verstanden wird. Uberlappen sich Gesprächsbeiträge extrem - wie es in der Alltagskommunikation vorkommt - ist dies für Treffer, die im Detail analysiert werden sollen, ebenfalls keine gute Voraussetzung. Solche Fälle, um hier nur zwei Beispiele zu nennen, müssen dann für Detailanalysen ausgeschlossen werden. 
Element vor Ahnung ( $n=275)$

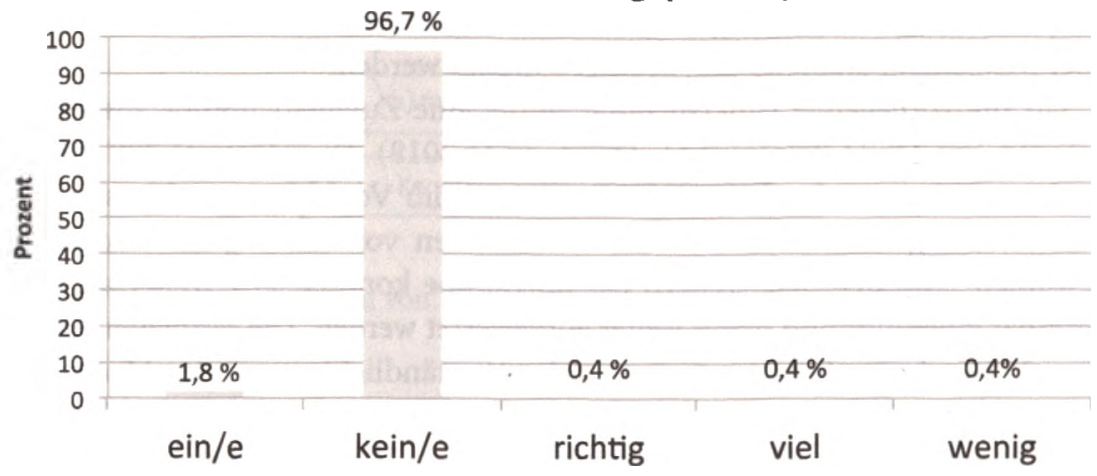

Abb. 2: Element vor Ahnung (FOLK)

Im Tool "Lexical Explorer" ${ }^{\text {14 }}$, das im Rahmen vom LeGeDe-Projekt entwickelt wurde und in dem über den gesamten Datensatz von FOLK quantitative Korpusdaten mit Hilfe von Häufigkeitstabellen bezüglich der Verteilung von Wortformen, Kookkurrenzen und Metadaten erforscht werden können, kann zur Verteilung der Bigrams mit Ahnung an 2. Position das Bild der Stichprobe auch über die Gesamtmenge an Vorkommen von Ahnung in FOLK bestätigt werden (vgl. Tab. 2). Die oben genannten Elemente viel, wenig und richtig- sind so ist in Tab. 2 abzulesen - Einmaltreffer im gesamten Korpus.

14 Lexical Explorer: URL: https://www.owid.de/lexex/, [letzter Zugriff; 02.08.2019]. 
Tab. 2: Bigrams zu Ahnung in FOLK (vgl. die Ergebnisse im "Lexical Explorer")

\begin{tabular}{|c|c|c|}
\hline normalisierte ${ }^{15}$ Form 1 & normalisierte Form 2 & (absolute) Frequenz \\
\hline roine & Ahnun: & 555 \\
\hline eine & Ahnung & 12 \\
\hline aein & Ahn & 2 \\
\hline wenig & Ahnung & 2 \\
\hline der & Ahni: & 1 \\
\hline schon & Ahnung & 1 \\
\hline Flatipe & Ahnun & 1 \\
\hline Management $^{16}$ & Ahnung & 1 \\
\hline Jiel & Ahn & 1 \\
\hline die & Ahnung & 1 \\
\hline hat & Ahnun & 1 \\
\hline keine & ahnung & 1 \\
\hline th & Ahnung & 1 \\
\hline
\end{tabular}

Ausgehend von diesen Ergebnissen ist die Verbindung keine Ahnung in der analysierten Stichprobe, aber auch in FOLK insgesamt, als die zentrale Verbindung in den gesprochen-sprachlichen Daten anzusehen.

(ii) Die Verbindung keine Ahnung $(\mathbf{n}=266)$ ist in der Stichprobe syntaktisch in unterschiedlicher Weise eingebettet. Es liegen $21 \%$ der Fälle vor (vgl. Abb. 3a), in denen keine Ahnung in eine größere syntagmatische Einheit integriert ist: jemand hat keine Ahnung [von]. Darunter ist eine Vollform (66\%) oder eine verkürzte Form (34\%) - also mit allen oder nicht allen Argumenten der Strukturmustereinheit - in den Daten der Stichprobe nachgewiesen (vgl. Abb. 3b), wie in den Bsp. (5) und (6) [Vollform] sowie (7) [verkürzte Form] illustrierend zu sehen ist.

15 In der DGD wird zwischen vier Ebenen der Annotiation von Zeichenketten unterschieden: einer transkribierten Ebene (z.B. kannscht), einer nomalisierten Ebene (z.B. kannst), der Lemma-Ebene (z.B. können) und der POS-Ebene, also der Part-of-SpeechEbene (z.B. VMFIN = Modalverb, finit). Die Bigrams in Tab. 2 zeigen die Elemente in normalisierter Form.

16 Das Element „Management" sticht auf den ersten Blick aus der Listung etwas heraus. Bei diesem Vorkommen handelt es sich um einen Äußerungskontext, in dem, ausgehend von einem Strukturmuster jemand hat Ahnung von etwas, die Präpositionalphrase mit von vorgezogen ist (0924: NG [((schmatzt) $\left.)^{\circ} \mathrm{h}\right]$ hat der mehr so vom management ahnung oder auch (.) vom (.) p; [FOLK_E_00293_SE_01_T_05_DF_01]). 
(5) FOLK_E_00257_SE_01_T_01_DF_01

0711 AA ich hab im internet halt nachgeschaut (.) äh woah des war ne

$0712 \quad(0.4)$

0713 AA schwierige phase wir $[\mathrm{kli}] \mathrm{ch}$ weil ganz alleine ich hab ja keine ahnung gehabt ${ }^{\circ h}$ un da hab ich halt im internet nachgeschaut überhaupt $\mathrm{mal}{ }^{\circ} \mathrm{hh}$ (.) was da so alles gibt und wohin ich gehör sozusagen welche kategor[ie]

$0714 \quad \mathrm{ZY} \quad[\mathrm{hmhm}]$

0715 ZY $[\mathrm{hm}](.) \mathrm{hmhm}($.

0716 AA ich gehör un was ich da machen kann welche unis mich annehmen mit diese[r prü] fung [und] hin und her ${ }^{\circ h h h}$

(6) FOLK_E_00059_SE_01_T_01_DF_01

0390 MF bis hin zu er hat_s (.) es hat ihn überhaupt nich interessiert ${ }^{\circ} \mathrm{h}$ was ich mir aber eigentlich eher (.) nich vorstellen kann weil in gewissermaße sind ja alle betroffen also wahrscheinlich schon ne neutrale stellung un er hat das auch beobachtet ${ }^{\circ} \mathrm{h}$ aber er hat sich dann nie äh berufen gefühlt ${ }^{\circ} \mathrm{h}$ da einzugr[eifen also]

$0391 \mathrm{HN}$ [ ${ }^{\circ} \mathrm{h}$ bis] hin zu sehr abwegich[en be]merkungen ein pas[tor auf] der alb ${ }^{\circ} \mathrm{hh}$ äh der eh keene ahnung hat [von] politik ${ }^{\circ} \mathrm{h}$ und demzufolge sich ${ }^{\circ} \mathrm{h}$ [direkt zurück] gezogen hat [ ${ }^{\circ h h h]}$

(7) FOLK_E_00218_SE_01_T_01_DF_01

$1065 \mathrm{P} Z \quad{ }^{\circ} \mathrm{h}($.$) keine ahnung was wir jetzt verschieben$

$1066 \quad(2.11)$

$1067 \mathrm{PZ}$ ohh wir (.) holen $\mathrm{h}^{\circ}$

$1068 \quad(0.84)$

1069 PZ 'hh jetzt mal des regal aus der ecke da raus weil da kommt jetzt eh keins hin ${ }^{\circ} \mathrm{h}$ wir schieben jetz des regal komm ma mit $h^{\circ}($.$) da rüber$

Die verkürzte Form (vgl. Bsp. (7) „keine ahnung was wir jetzt verschieben“) ist in den Daten besonders in der Variante keine Ahnung, was/wie/warum/wo zu finden, also im Strukturmuster jemand hat keine Ahnung (von etwas) ohne Subjekt und Hilfsverb, aber mit angeschlossenem, durch w-Adverbien eingeleitetem Nebensatz.

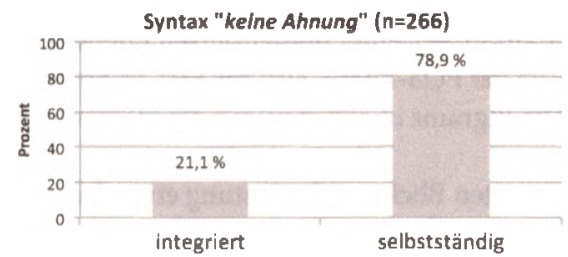

Abb. 3a: Syntax keine Ahnung (FOLK)

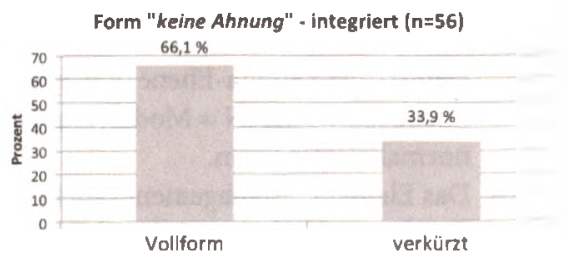

Abb. 3b: Form keine Ahnung [integriert] (FOLK) 
Der sehr viel größere Teil (78,9 \%) von keine Ahnung tritt als selbstständige Einheit im Gesprächsverlauf auf (vgl. Abb. 3a). In Bsp. (8) ist ein solches Vorkommen dargestellt. Hier fungiert die Einheit keine Ahnung als eigenständiger Turn und besitzt als ganze Einheit eine kommunikative Funktion.

(8) FOLK_E_00175_SE_01_T_01_DF_01

\begin{tabular}{|c|c|c|}
\hline $\begin{array}{l}0203 \\
0204\end{array}$ & $\mathrm{CW}$ & $\begin{array}{l}\text { in doktor ich a ge d da da schneiden wir irgendwann ein lammherz } \\
(0.48)\end{array}$ \\
\hline 0205 & OW & $\mathrm{i}[\mathrm{h}]$ \\
\hline 0206 & CW & [(und) gucken was] drinne is \\
\hline 0207 & MW & [a ha] \\
\hline 0208 & SW & [ouh] \\
\hline 0209 & & $(0.57)$ \\
\hline 0210 & SW & und was könnte drin sein \\
\hline 0211 & FW & ha [ha a] oh ha ha \\
\hline 0212 & $\mathrm{CW}$ & [keine ahnung] \\
\hline
\end{tabular}

Die $78,9 \%$ Vorkommen von keine Ahnung als selbstständige Einheit $(n=210)$ wurden außerdem noch danach untersucht, an welcher Stelle die Einheit im Gesprächskontext von den SprecherInnen eingesetzt wird. Es wird bei den Analysekriterien unterschieden zwischen "alleinstehend", „vorangestellt" (145 LZ keine ahnung mia kommt jetz irgendwie vor es wär alles andersch; FOLK_E_00268_SE_01_T_01_DF_01), „parenthetisch“ (892 VB ja da kriegst keine ahnung s fünffache in der stund wie als ergo; FOLK_E_00318_ SE_01_T_01_DF_01) oder "nachgestellt“ (114 LE [so gr] ün bl[au oder s]o so [türkis] keine ahnung; FOLK_E_00329_SE_01_T_06_DF_01).

Mit 46,2 \% kommt die Einheit am häufigsten alleinstehend vor (vgl. Abb. 4), so wie in Bsp. (8) bereits gezeigt wurde. In diesen Kontexten stellt die Einheit meist eine eigenständige Äußerung in Form einer Reaktion (z.B. einer Antwort auf eine Frage) auf eine vorausgegangene Äußerung dar. In vielen Fällen liegt dann eine responsive Verwendung vor.

\section{Form "keine Ahnung" - selbstständig ( $\mathrm{n=210)}$}

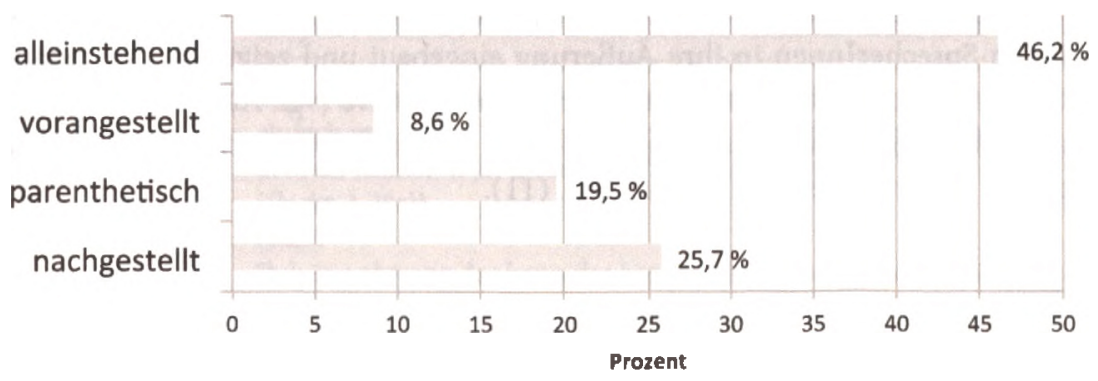

Abb. 4: Form keine Ahnung [selbstständig] (FOLK) 
Außerdem können in den Daten der Stichprobe unter den Vorkommen von keine Ahnung als selbstständige Einheit $(\mathrm{n}=210)$ nicht-responsive Fälle $(\mathrm{n}=127)$ ausgemacht werden (vgl. Abb. 5).

Orientierung "keine Ahnung" - selbstständig / nicht-responsiv (n=127)

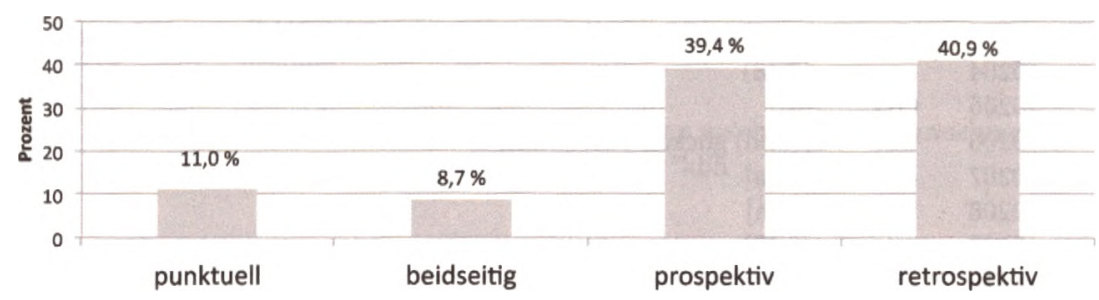

Abb. 5: Orientierung keine Ahnung [selbstständig / nicht-responsiv] (FOLK)

Ist keine Ahnung in einem Gesprächskontext z.B. einer Äußerung nachgestellt (25,7 \%), liegt meist eine nicht-responsive Verwendung vor (vgl. Abb. 4). Mit der Einheit keine Ahnung beenden SprecherInnen ihre eigene Äußerung. Die Orientierung ist dabei vor allem auf die zuvor getätigte Äußerung gerichtet und keine Ahnung kann damit als retrospektiv eingestuft werden (vgl. Abb. 6d und Bsp. (9)).

(9) FOLK_E_00084_SE_01_T_01_DF_01: nicht-responsiv/nachgestellt/retrospektiv 0196 EG (.) ja erst ma eigentlich nur bis sonntag aber wahrscheinlich wird er noch mal zwei [wochen krankgeschrieb]

0197 FR [hä das muss aber] läng[er oder] was

0198 EG [ja]

0199 EG ja das (.) war halt nur im krankenhaus äh ich weiß auch nich das hat [wahrscheinlich irgendwelch]e (.) büro[kratischen gründe] (.) keine ahnung (.) ((zieht die Nase hoch))

Bei parenthetisch eingeschobenem keine Ahnung (19,5\%) zeigt die Stichprobe (vgl. Abb. 4) zumeist nicht-responsive Verwendungen. Die Einheit wird von den SprecherInnen in ihre Äußerung eingebaut und zeigt von der inhaltlichen Orientierung her insbesondere eine prospektive (vgl. Abb. 6c) oder auch eine beidseitige Ausrichtung (vgl. Abb. 6b); vgl, zur prospektiven Ausrichtung Bsp. (10) und zu einer beidseitigen Bsp. (11). 

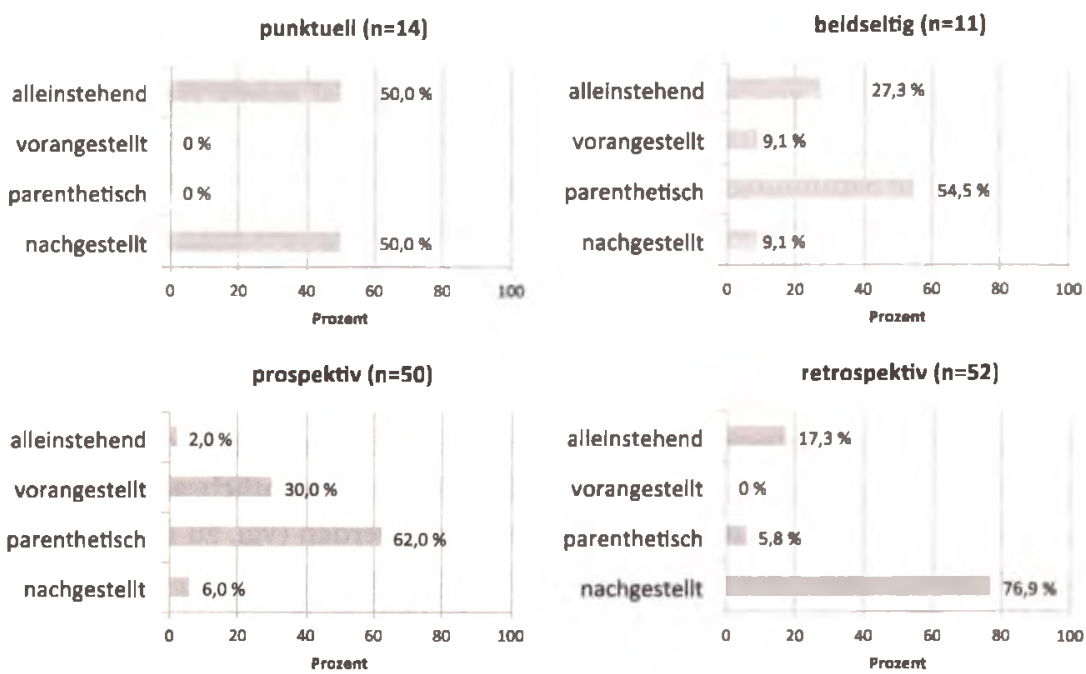

Abb. 6a-d: Orientierung keine Ahnung - selbstständig/nicht-responsiv $(n=127)$ (a. punktuell, b. beidseitig, c. prospektiv, d. retrospektiv)

(10) FOLK_E_00253_SE_01_T_01_DF_01: nicht-responsiv/parenthetisch eingeschoben/prospektive Ausrichtung

0501 FN j[a so soll_s s]ein ${ }^{\circ} \mathrm{h}$ [ja] ja nürnberg is is a

0502 ML $[(($ lacht $))]$

0503 ML $[\mathrm{hm}]$

$0504 \quad(0.32)$

0505 FN is groß ja des stimmt abe[r $\mathrm{i}] \mathrm{ch}$ mein keine ahnung so nach fast fünfunzwanzig jahrn kennt man dann halt trotzdem irgendwie viel

(11) FOLK_E_00330_SE_01_T_03_DF_01: nicht-responsiv/parenthetisch eingeschoben/beidseitige Ausrichtung

0347 RK sehr sehr viel wegnehmen wenn die cecilie einfach die ganze zeit nur ein ja un amen äh tochter is die dann im endeffekt ${ }^{\circ} \mathrm{h}$ ich mein (.) des is schon noch irgnwo $n$ intressanter punkt weil wenn du jetz $n$ realen abend hast wieso gehst du dann mit diesem mann mit nimmt der dich einfach mit un das is dann halt der nächste bei dem du ja un amen präbeln kannst ${ }^{\circ} \mathrm{h}$ ja ${ }^{\circ} \mathrm{h}$ vater (.) er is mein (.) freund

$0348 \mathrm{JH} \quad(($ lacht, 1.28s))

$0349 \quad(0.29)$

0350 RK oh keine ahnung das kann des kann auch sein aber des würd ich halt dir überlassen wie du des gerne $\mathrm{sp}$ [ielen würde]st (.) also ich würd vor all dingen des gerne ähm innerhalb der beziehun[gen sich entwick] eln lass[en weil_s sehr schwierig] so was einfach ${ }^{\circ} \mathrm{h}$ 


\subsection{Ahnung in DEREKo}

Für die Analyse von geschrieben-sprachlichem Material wurde das „Deutsche Referenzkorpus" (DEREKo) gewählt. Bei diesem Korpus handelt es sich um die weltweit größte Sammlung deutschsprachiger Korpora, die als Basis für linguistische Forschung herangezogen werden kann. Mit Stand März 2019 (18.03.2019) umfasst DeReKo 43 Milliarden Wörter, die aus geschriebenen deutschsprachigen Texten aus der Gegenwart und der neueren Vergangenheit stammen. Die Texte weisen ein großes Spektrum an Textsorten auf: Neben u.a. belletristischen, wissenschaftlichen und populärwissenschaftlichen Texten umfasst DEREKo eine sehr große Zahl an Zeitungs- sowie Zeitschriftentexten und wird kontinuierlich gepflegt und ausgebaut. In den ausschließlich urheberrechtlich abgesicherten Daten kann zurzeit über COSMAS II recherchiert werden (vgl. zu DEREKo u.a. Kupietz/Keibel 2009, Kupietz et al. 2010, Kupietz et al. 2018).

Bei der Stichprobe, die für die vorliegende Untersuchung gezogen wurde, handelt es sich um eine Zufallsstichprobe ${ }^{17}$. Die Suchabfrage mit dem \&-Operator (\&Ahnung) ermöglicht die Abfrage nach allen Wortformen. Kompositabildungen wurden für die Suchabfrage ausgeschlossen. Die Stichprobe umfasst mit dieser Abfrage insgesamt 300 Treffer. In nur $3 \%$ der 300 Fälle ist die Pluralform verzeichnet (vgl. Bsp. (12)-(14)).

(12) DeREKo: Berliner Morgenpost, 27.11.2014, S. 2; Ahnungen und Andeutungen Statt Gewissheiten streut Benson nur Ahnungen und Andeutungen.

(13) DeReKo: Die Presse, 22.07.1997, Ressort: Kultur; Von Pavarottis Beichte und Zwölfmaltonklugen

Düstere Ahnungen steigen auf.

(14) DeReKo: Protokoll der Sitzung des Parlaments Landtag Schleswig-Holstein am 28.08.1997. 38. Sitzung der 14. Wahlperiode 1996-2000. Plenarprotokoll, Kiel, 1997

Trotzdem, Sie haben von Ahnungen gesprochen. Ich meine, in der Wirtschaft von Ahnungen zu sprechen, ist nicht ganz opportun.

Ganz bewusst wurden bei der Ziehung keine Ausschlusskriterien von Quellen formuliert. Von Zeitungsquellen über Belletristik, von Wikipedia-Diskussionsseiten bis zu schriftlichen Aufzeichnungen von Bundestagsreden ist in der Stichprobe eine große Bandbreite an Quellen enthalten. So lässt sich, ohne vorherige Hypothese und Einschränkungen, ablesen, in welchen Quellenarten Ahnung in der Stichprobe vorkommt (vgl. Abb. 7).

17 Für die Unterstützung bei der Ziehung der Stichprobe aus DeReKo und der Datenaufbereitung, die die weiteren Kodierungen und Analysen sehr erleichtert hat, danke ich Rainer Perkuhn (IDS, Korpuslinguistik). 


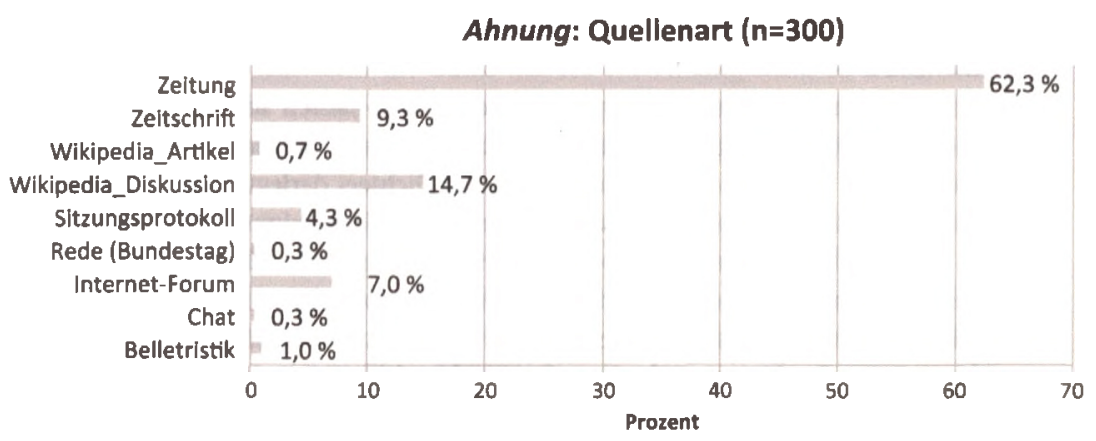

Abb. 7: Ahnung (Quellenart) (DEREKo)

Mit $62 \%$ ist ein sehr hoher Anteil an Vorkommen von Ahnung in Zeitungstexten verzeichnet. Der Anteil an Zeitungsquellen in DeReKo insgesamt ist bezogen auf das gesamte Korpus der weitaus größte Anteil. Somit spiegelt das Ergebnis auch ein Stück weit die Zusammensetzung der Daten in DeREKo insgesamt wider. Die nächst häufigsten Quellen, in denen Ahnung nachgewiesen ist, sind in der Stichprobe die Wikipedia-Diskussionsseiten (14,7\%), Zeitschriften $(9,3 \%)$ und mit $7 \%$ Internet-Foren-Einträge. Interessant dabei ist, dass insbesondere die Texte der Wikipedia-Diskussionsseiten sowie die der Internet-Foren-Einträge eher einem „Duktus der Mündlichkeit“ (Schlieben-Lange 1983: 81) zugerechnet werden können. Zu diesem Ergebnis der Quellenart können damit noch zwei weitere Fragen gestellt werden: 1. Lassen sich insbesondere in den Zeitungstexten Auffalligkeiten zur Verwendung von Ahnung beobachten? und 2. Welche Verwendungsbesonderheiten von Ahnung lassen sich in Quellenarten ausmachen, die einem „Duktus der Mündlichkeit" zugerechnet werden können? Um diesen beiden Fragen nachzugehen, kann zunächst ein Blick auf die Textarten der gesamten Stichprobe geworfen werden (vgl. Abb. 8). Narrative Textpassagen, in denen Ahnung vorkommt, sind mit 33,3\% am stärksten in den 300 Treffern vertreten. Bsp. (15) und (16) illustrieren typische Vorkommen.

(15) DeReKo: Braunschweiger Zeitung, 30.10.2010; So funktionieren die Experimente

Physik macht Spaß. Auch dann, wenn man gar nicht so genau weiß, was da eigentlich gerade passiert. Aber - ein bisschen Wissen hat jeder, und dann auch eine gewisse Ahnung, wie das alles zusammenpasst. Zum 5-jährigen Bestehen des Phaeno stellen die WN in jeder Woche zwei Phaeno-Experimente vor, die jeweils von Phaeno-Besuchern erklärt werden. 
(16) DEREKo: Wilkenloh, Wimmer: Hätschelkind, [Kriminalroman], - Meßkirch, 14.04.2011

In der Husumer Rundschau hatte er in den letzten Jahren schon öfter Artikel über die Theorie eines gewissen Ruppert Wraage gelesen, der immer wieder seine Meinung von einem unentdeckten Roman des Dichters darlegte. Doch die bissigen Kommentare der hiesigen Experten begleiteten jede seiner Veröffentlichungen im Lokalblatt nur mit Hohn und Spott. Er selber hatte die Vorstellung von einem Roman seines Lieblingsdichters immer spannend gefunden. Und jetzt gab es diesen Roman höchstwahrscheinlich wirklich! Edda hatte offensichtlich keine Ahnung, was ihr da so unverhofft in die Hände gefallen war.

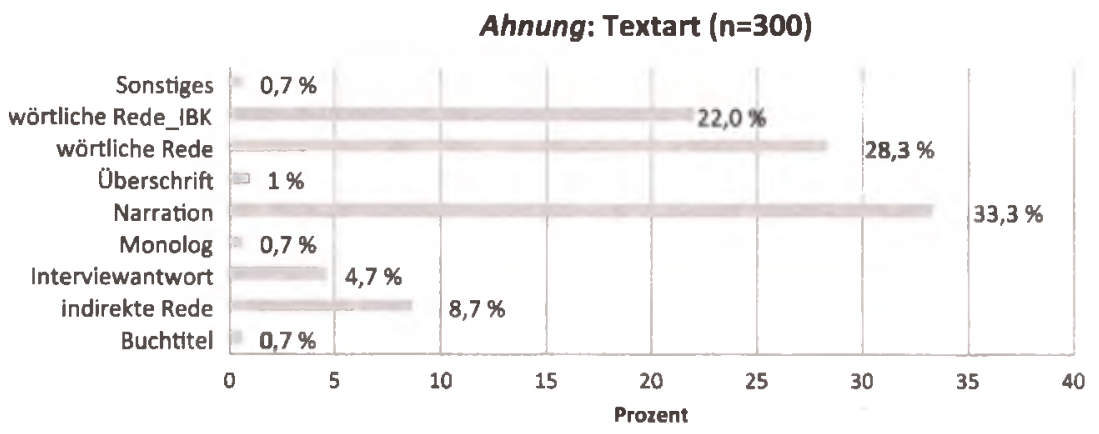

Abb. 8: Ahnung (Textart) (DeReKo)

Daneben ist in Bezug auf die Textart, in der Ahnung vorkommt, wörtliche Rede $(28,3 \%)$ und indirekte Rede $(8,7 \%)$ in der Stichprobe auszumachen (vgl. Abb. 8). Mit $22 \%$ ist auch eine Kategorie "wörtliche Rede_IBK“ recht häufig vertreten. Hierunter sind Textpassagen gebündelt, in denen Ahnung in quasi "fiktiver" wörtlicher Rede in Texten vorkommt, die aus dem Bereich der internetbasierten Kommunikationen stammen (= IBK, z.B. Wikipedia-Diskussionsseiten, Internet-Foren-Beiträge, Chat). Die Bsp. (17) und (18) illustrieren das Vorkommen von Ahnung auf einer Wikipedia-Diskussionsseite sowie aus einem Chatprotokoll (siehe dazu auch weiter unten in diesem Abschnitt).

(17) DEREKo: http://de.wikipedia.org/wiki/Diskussion:Ausbildungsskala: Wikipedia, 2011. Diskussion: Ausbildungsskala, In: Wikipedia.

Habe die sechs betroffenen Artikel mit den Informationen aus diesem hier aufgepeppt, wo sinnvoll. Ich finde, das ist ein Gewinn für alle acht Artikel und hoffe, dass Ihr das auch so seht und die Artikel durch die Sichtung kommen. --212.77.163.106 13:04, 20. Jan. 2010 (CET) 
Da hab ich keine Bedenken:). Vielleicht sollten wir in die einzelnen Artikel noch eine Box hereinsetzen, die Links auf die Ausbildungsskala und ihre einzelnen Punkte enthält $\rightarrow$ zur Not auch eine Navigationsleiste, wobei mir die prominent oben rechts untergebrachte Box deutlich lieber ist. --82.113.121.98 13:26, 20. Jan. 2010 (CET)

Puh... klingt gut, aber keine Ahnung, wie man sowas macht. Die Idee gefällt mir aber echt gut! --212.77.163.101 13:36, 20. Jan. 2010 (CET)

(18) DEREKo: 2101002_tour05 (ARD), [Chat-Logfile], In: Dortmunder Chatkorpus, Plauder-Chats

wie geht das denn?

$04: 35: 13$

keine ahnung

$04: 35: 22$

vermutlich falsch gedrückt

Mit Kategorien wie „Überschrift“, „Narration“, „Buchtitel“ und auch eher nicht klar bestimmbare Fälle unter der Kategorie „Sonstiges“ (vgl. in Tab. 3 = A) können in Bezug auf die Textart Passagen mit Ahnung ausgemacht werden, die dem „Duktus der Schriftlichkeit“ zuzurechnen wären. Demgegenüber weisen die Vorkommen von Ahnung in den Kategorien „wörtliche Rede(_IBK)“, „Mono-

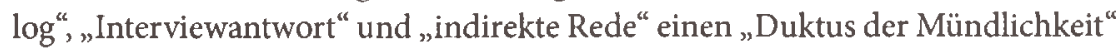
auf (vgl. in Tab. 3 = B). Mit 64,3\% in Gruppe B weist Ahnung ein sehr viel höheres Vorkommen in Textarten mit Mündlichkeitsduktus auf als im Vergleich zu Gruppe A mit 35,7\%.

Tab. 3: Ahnung Textarten zusammengefasst in Gruppe A und B

\begin{tabular}{llc}
\hline Gruppe & Kategorie & Prozent \\
\hline A & Úberschrift, Narration, Buchtitel, Sonstiges & $35,7 \%$ \\
B & wörtliche Rede(_IBK), Monolog, Interviewantwort, indirekte Rede & $64,3 \%$ \\
\hline
\end{tabular}

Zur weiteren Analyse der Daten zu Ahnung aus DEREKo soll - ähnlich wie bei der Datenanalyse der Treffer aus FOLK - erneut auf zwei Aspekte genauer eingegangen werden: (i) das kombinatorische Potenzial von Ahnung (insbesondere bezogen auf das direkt links vorkommende Elemente in Form von Artikeln, Adjektiven, Zahladverbien etc.), (ii) die Betrachtung der Kombination keine Ahnung als eine zentrale Verbindung unter verschiedenen Gesichtspunkten (syntagmatische Einbettung, Formbesonderheiten sowie die Stellung im Äußerungskontext).

(i) Die Betrachtung des Elementes im direkten linken Kontext von Ahnung in der Stichprobe zeigt eine klare Tendenz: Das Determinativum kein- ist mit 
$67 \%$ auch in diesen Daten der Spitzenreiter in der Liste der in der Stichprobe vorkommenden Kombinationen (vgl. Abb. 9).

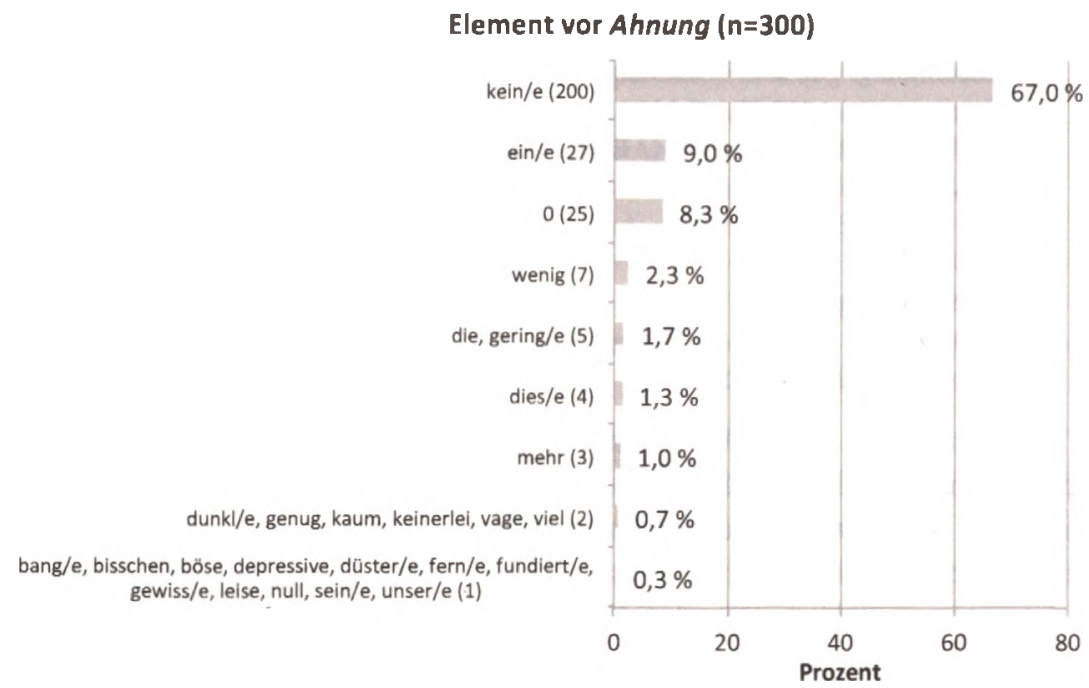

Abb. 9: Element vor Ahnung (DeReKo)

In $A b b .9$ kann neben dem prozentualen Wert auch rechts neben dem jeweiligen Element abgelesen werden, wie oft das Element absolut in der Stichprobe $(\mathrm{n}=300)$ vorkommt. Der indefinite Artikel ein-ist nach kein- mit $9 \%$ Vorkommen das nächst häufigste Elemente vor Ahnung. In 8,3\% der Fälle steht kein attribuierendes Element vor Ahnung. Die weiteren Elemente wie wenig (2,3\%), gering/e (1,7 \%) etc. kommen sieben oder weniger als sieben Mal in der Stichprobe vor. Das Spektrum an verschiedenen Möglichkeiten ist aber schon auf die Stichprobe $(n=300)$ betrachtet sehr hoch. In den gesprochen-sprachlichen Daten ist das Spektrum viel eingeschränkter (vgl. Abb. 2 in Abschnitt 3.2).

Eine Kookkurrenzanalyse über alle öffentlichen Korpora des Archivs W in DEREKo ${ }^{18}$ mit den Suchparametern „1 Wort links, 0 Wörter rechts“ von Ahnung und der Hinzunahme von Funktionswörtern, zeigt unter den TOP-20 der Bigrams, dass das Determinativum kein- auch über die Gesamtmenge das häufigste Wort vor Ahnung ist (vgl. Tab. 4).

18 Die Kookkurrenzanalyse wurde am 13.04.2019 über DEREKo-2019-I durchgeführt. 
Tab. 4: TOP-20 der Kookkurrenzen zu Ahnung (1 Wort links, 0 Wörter rechts) (DEREKO) ${ }^{19}$

\begin{tabular}{rrrll}
\hline Nr. & LLR $^{20}$ & Abs. Frequenz & Kookkurrenz & syntagmatisches Muster \\
\hline 1 & 719308 & 75534 & keine & keine Ahnung \\
2 & 239177 & 20372 & Keine & Keine Ahnung \\
3 & 23228 & 4099 & wenig & wenig Ahnung \\
4 & 20229 & 1630 & geringste & geringste Ahnung \\
5 & 9949 & 639 & leiseste & leiseste Ahnung \\
6 & 6608 & 782 & null & null Ahnung \\
7 & 5284 & 1898 & viel & viel Ahnung \\
8 & 3627 & 485 & leise & leise Ahnung \\
9 & 3619 & 503 & böse & böse Ahnung \\
10 & 3562 & 301 & blasse & blasse Ahnung \\
11 & 3437 & 395 & vage & vage Ahnung \\
12 & 2974 & 473 & keinerlei & keinerlei Ahnung \\
13 & 2750 & 222 & ungefähre & ungefähre Ahnung \\
14 & 2560 & 360 & dunkle & dunkle Ahnung \\
15 & 2475 & 278 & düstere & düstere Ahnung \\
16 & 2424 & 319 & ne & ne Ahnung \\
17 & 2413 & 1223 & Eine & Eine Ahnung \\
18 & 1350 & 363 & bisschen & bisschen Ahnung \\
19 & 1328 & 201 & bösen & bösen Ahnungen \\
20 & 1275 & 1581 & mehr & mehr Ahnung \\
\hline
\end{tabular}

Auffällig in dieser Liste ist das doppelte Vorkommen von keine/Keine (Nr. 1 und 2). Die dazugehörigen „Keywords in context“ (KWIC) zeigen, dass es sich bei den Keine-Fällen zumeist um „Keine Ahnung"-Äußerungen in wörtlicher Rede handelt. Darunter fallen also vor allem selbstständige Äußerungen, die als eigenständige kommunikative Einheit fungieren (vgl. Bsp. (19)).

(19) DeReKo: St. Galler Tagblatt, 24.04.2010, S. 45; Orchester aus Alltagsgegenständen

Aus den Klavier-Einzelteilen und anderem Bastelmaterial stellen die Schüler neue Instrumente her, die aus einem Klangkörper und Saiten bestehen. Ein Knabe zieht

19 Die Tab. 4 zeigt die ersten 20 Kookkurrenzen beginnend mit der häufigsten unter Angabe des LLR-Wertes, der absoluten Frequenz, der jeweiligen Kookkurrenz und des syntagmatischen Musters.

20 "LLR" ist die Abkürzung für "log likelihood ratio " und steht als Maßzahl für die Abweichung des normalen Verhaltens vom beobachteten. 
eine Klaviersaite durch ein Plastikkörbchen. Ein helles Surren entsteht. Wie das Instrument heisst? "Keine Ahnung.» Hauptsache, es tönt.

Auch das Element Nr. 16 (ne) ist in den Daten in speziellen Verwendungen zu finden: Zumeist kommt die verkürzte Form des indefiniten Artikels (eine) in Verbindungen vor, die allgemein im Strukturmuster jemand hat ne Ahnung (von etwas) zu beschreiben sind, und die konkret in Verwendungen wie von nichts/nix (')ne Ahnung, Hast du 'ne Ahnung! oder hast du 'ne Ahnung, was/wo bzw. (hat) jemand ne Ahnung, was, wo, ob vorkommen. Die Variante unter Nr. 17 (Eine) ist ebenfalls auffällig, weil sie an Satzanfängen zu finden ist (abstrahiert: Eine Ahnung von etwas bekommt jemand/vermittelt etwas), in Zwischenüberschriften (abstrahiert in der Form: Eine Ahnung von etwas) vorkommt, oder in Buchtiteln.

(ii) Die Analyse des Elementes vor Ahnung hat das sehr hohe Vorkommen von kein- in der Stichprobe gezeigt. Im Folgenden soll dieser Kombination noch etwas genauer nachgegangen werden. Dazu werden als Ausgangspunkt die Treffer von keine Ahnung ( $\mathrm{n}=200$ ) aus der Stichprobe in Bezug auf das Vorkommen in den verschiedenen Textarten betrachtet (vgl. Abb. 10). In wörtlicher Rede $(34,5 \%)$ kommt keine Ahnung am häufigsten vor. Der Charakter dieser Verbindung als eine, die einen mündlichen Duktus aufzuweisen scheint, wird mit diesem hohen Vorkommen unterstrichen.

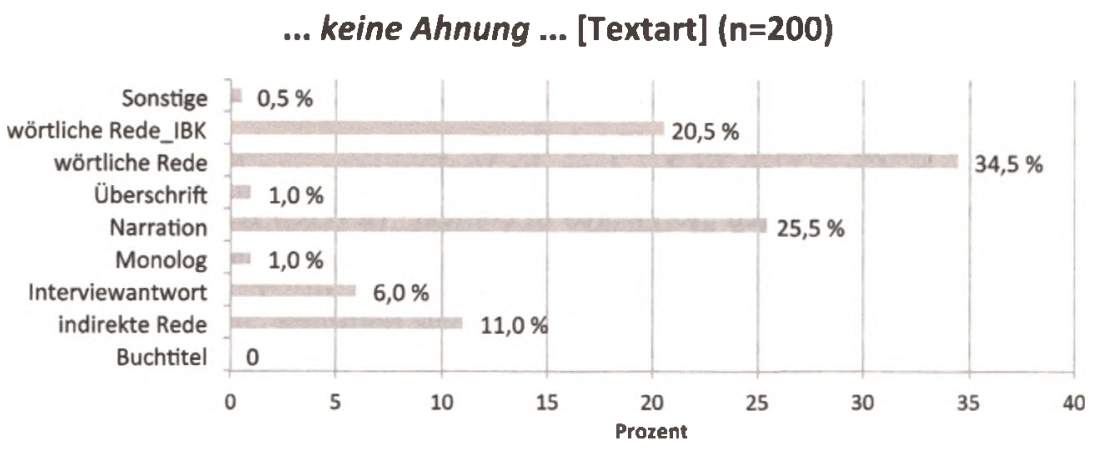

Abb. 10: Verbindung keine Ahnung [Textart] (DEREKo)

Unter den Fällen von keine Ahnung (69 Treffer) in wörtlicher Rede (vgl. Abb. $10)$ ist die Verbindung in den meisten Fällen (61) in einen syntagmatischen Kontext eingebettet (vgl. Bsp. (20)). Bei den Vorkommen von nicht-eingebettetem und damit von selbstständigem Vorkommen der Verbindung handelt es sich um alleinstehende Äußerungen, die zumeist als Antwort auf eine Frage 
(also in responsiver Funktion) eingesetzt werden (vgl. Bsp. (21) bzw. (22). Bsp. (22) zeigt dabei die Niederschrift einer tatsächlichen wörtlichen Rede von einer Äußerung im Bundestag).

(20) DEREKo: Niederösterreichische Nachrichten, 18.07.2013; Ärger wegen E-Mails Der Beschuldigte, ein 23-Jähriger aus dem Bezirk Horn, gab sich einsichtig. Er mache schon eine Therapie und akzeptiere nun das Beziehungsende. Außer den sieben geschickten E-Mails habe er keinen Kontakt zu der Ex-Freundin aus Sigmundsherberg mehr gesucht, beteuerte er. "Sie haben keine Ahnung, was Sie für ein Glück haben. Sieben Nachrichten reichen nach einem OGH-Entscheid zur Erfüllung des Tatbestandes Stalking nicht aus. Es müssen mindestens zehn Einzelhandlungen sein. Es ergeht ein Freispruch, aber nur aus rein rechtlichen Gründen", erklärte die Richterin.

(21) DeReKo: Nürnberger Zeitung, 13.02.2015, S. 22; Glücksangst // Gott und die Welt

Wir tauschten unsere Erfahrungen aus. Ein Gefängnisseelsorger sagte: „Weißt du, wovor die Gefangenen am meisten Angst haben?" Ich: „Keine Ahnung". Er: „Am meisten haben sie Angst vor dem Tag, an dem sie entlassen werden."

(22) DeReKo: Dückert, Thea: Arbeitsmarktpolitik. Rede im Deutschen Bundestag am 06.06.2002, Hrsg: Bundestagsfraktion Bündnis 90/DIE GRÜNEN

(Wolfgang Meckelburg [CDU/CSU]: Klassenkampf!)

(Beifall bei Abgeordneten des BÜNDNISSES 90/ DIE GRÜNEN und der SPD)

(Beifall beim BÜNDNIS 90/DIE GRÜNEN und bei der SPD - Ernst Hinsken [CDU/CSU]: Das ist doch Blödsinn hoch drei, was Sie hier sagen! Keine Ahnung!Wolfgang Meckelburg [CDU/CSU]: Sie sollten das Programm wirklich einmal lesen!)

(Beifall beim BÜNDNIS 90/DIE GRÜNEN und bei der SPD)

\section{Ahnung ... und darüber hinaus}

Die drei Bedeutungen von Ahnung (vgl. Abb. 1), die aus den Wörterbüchern abgelesen und im weitesten Sinne dem Bereich ,Epistemik' zugeordnet werden können, lassen sich grundsätzlich in den authentischen Beispielen wiederfinden (vgl. Abb. 11/12). Die Lesart b) (mitgeteiltes, erlebtes Wissen) ist dabei besonders häufig in den Daten, insbesondere bei FOLK, zu finden (vgl. z.B. Bsp. 4, 5, $6,7,15,16,17,20$ ). Die Lesart a) (vages (Vor-)Gefühl, Erwartung) ist eher in den DeReKo-Daten zu finden (vgl. z.B. Bsp. 12-14). Lesart c) (Kenntnis, durch Lernen erworben, vgl. Bsp. 2 und 3) ist in beiden Korpora vertreten, kommt im Verhältnis häufiger in den DEREKo-Daten vor. 


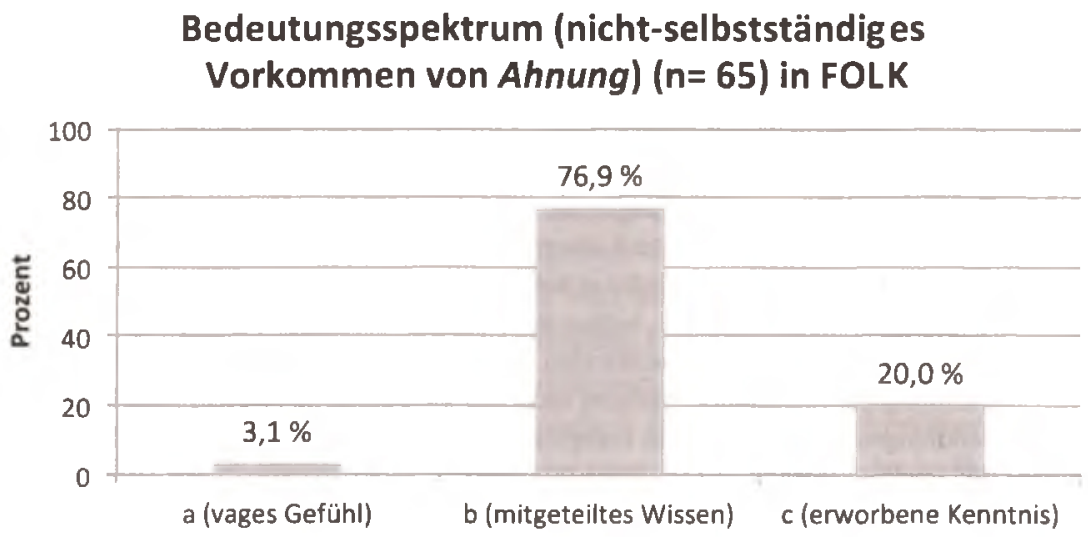

Abb. 11: Bedeutungsspektrum von Ahnung (nicht-selbstständiges Vorkommen) in FOLK
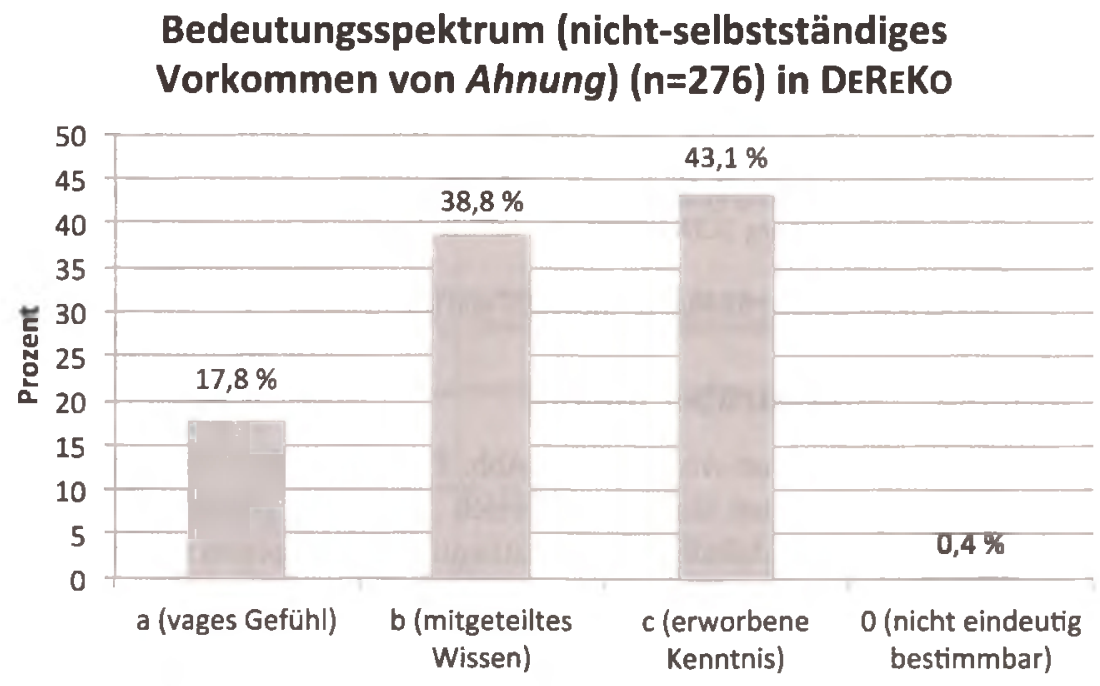

Abb. 12: Bedeutungsspektrum von Ahnung (nicht-selbstständiges Vorkommen) in DEREKo ${ }^{21}$

21 Bei dem nicht bestimmbaren Fall (0,4\% ist 1 Fall in den Daten) handelt es sich um einen Buchtitel, der in seiner Syntax sehr verkürzt ist. Daher ist die eindeutige Zuweisung einer Bedeutung hier nicht möglich. 
Zu dem Ergebnis gibt es, was die Interpretation betrifft, noch zwei kritische Anmerkungen:

1. Für die Betrachtung des Bedeutungsspektrums wurden diejenigen Fälle aus der Stichprobe ausgeschlossen, bei denen die Kombination keine Ahnung als selbstständige Einheit fungiert (vgl. Bsp. (8) aus FOLK oder Bsp. (19) aus DEREKo). Sicherlich ist in der Kombination in einigen Fällen noch eine Grundbedeutung von Ahnung enthalten, dies wäre aber eine eigene Betrachtungsweise. Bei Ausschluss dieser Kombination konnten in den FOLK-Daten lediglich 65 Fälle untersucht werden, auf deren Basis das Bedeutungsspektrum von Ahnung in relativem Verhältnis in Abb. 11 dargestellt ist. Wir können daraus auch schlussfolgern, dass in den FOLK-Daten die Kombination keine Ahnung als selbstständige Einheit im Verhältnis zum Vorkommen von Ahnung in eingebetteten Kontexten recht häufig vertreten ist. In der DEREKo-Stichprobe sind im Vergleich zu den FOLK-Daten lediglich 24 Fälle verzeichnet, in denen keine Ahnung als selbstständige Einheit vorkommt, sodass die Menge an analysierten Fällen (276) im Vergleich zu den FOLK-Daten sehr viel höher ausfällt.

2. Eine Umschreibung der Bedeutung ,vages Gefühl' trifft es bei den FOLKDaten partiell nicht ganz. Die Belege gehen teils eher in die Richtung,Vorstellung von etwas haben. Der Vergleichbarkeit wegen ist in Abb. 11 die Formulierung beibehalten. Die Analyse einer größeren Datenmenge könnte hier noch mehr Aufschluss geben.

Die Untersuchung hat gezeigt, dass das Determinativum kein- auffällig häufig vor Ahnung tritt (vgl. Abb. 2 und Tab. 2 sowie Abb. 9 und Tab. 4). In dieser Verbindung findet sich keine Ahnung in verschiedenen Ausprägungen, die dem Grundstrukturmuster jemand hat keine Ahnung (von etwas) zugeordnet werden können. Besonders typisch für den „Duktus der Mündlichkeit“ scheint die Verwendung von keine Ahnung als selbstständige Einheit. Auch in den DeREKoDaten finden sich solche Verwendungsweisen. Die Untersuchung konnte zeigen, dass hier der Anteil an Textarten, in denen der "Duktus der Mündlichkeit" auszumachen ist, besonders hoch ist - z.B. in Niederschriften von Reden, aber auch in Texten aus dem Bereich der internetbasierten Kommunikation wie z.B. auf Wikipedia-Diskussionsseiten. Insbesondere der Gebrauch von keine Ahnung als selbstständige Einheit weist interessante Facetten an Funktionen auf. Neben einer teils noch wiederzuerkennenden Verbindung zum epistemischen Gehalt von Ahnung in der Grundbedeutung (meist sind Anteile von c), erworbenes Wissen' zu erkennen) fächert sich ein Spektrum an kommunikativen Funktionen auf, das in den untersuchten Wörterbüchern nicht angemessen abgebildet 
ist. Einzig das PONS-DaF listet als Beispiel auf: Weißt du, wo mein Schlüssel ist? Keine Ahnung! (vgl. Tab. 1). Das in der gesprochenen Sprache parenthetisch eingeschobene oder einer Äußerung vor- und nachgestellte keine Ahnung wird an keiner Stelle erwähnt. Die Arbeiten von Bergmann (2017), König/Stoltenburg (2013) oder Eichinger (2018) zeigen keine Ahnung u.a. als etcetera-Formel, als epistemischen Unschärfemarker und/oder als Gliederungsmarker. Die Nennung dieser nur drei Funktionen will andeuten, welche funktionale Tiefe die Verbindung insbesondere im Interaktionskontext besitzt. Da die Wörterbücher (vgl. Abschnitt 3.1) das Bedeutungs- bzw. Funktionsspektrum nicht auf großen gesprochen-sprachlichen Korpora erarbeitet haben, verwundert es nicht, dass die Verbindung keine Ahnung als insbesondere zentrale Einheit im Gesprochen dort mit ihrem Funktionsspektrum nicht vollständig abgebildet ist.

Das in Abschnitt 1 erwähnte LeGeDe-Projekt ${ }^{22}$ am IDS möchte diese Lücke ansatzweise schließen. In einem Modul 1 geben Erläuterungen zur Grundbedeutung des Lexems Ahnung Auskunft über das Bedeutungsspektrum, wie sich dieses in einer systematisch definierten Stichprobe aus FOLK dargestellt. In einem Modul 2 wird die Verbindung keine Ahnung näher betrachtet und es werden vor allem interaktionslinguistische Analysen auf Basis der gezogenen Stichprobe durchgeführt. Im Ergebnis erhalten Nachschlagende Informationen zu Ahnung in den „Bedeutungen“ (linker Bereich) sowie zu keine Ahnung mit seinen „Funktionen im Gespräch" (rechter Bereich) (vgl. dazu Abb. 13). Authentische Belege aus FOLK (Transkripte sowie Audiomaterial) illustrieren in Modul 1 und in Modul 2 die Wörterbuchangaben (vgl. dazu Abb. 14).

22 Die Ausarbeitung zu Ahnung (zu GrundbedeutungeI der Verbindung keine Ahnung) kann im LeGeDe-Prc https://www.owid.de/legede/, [letzter Zugriff: 12.02.2ULU]). 


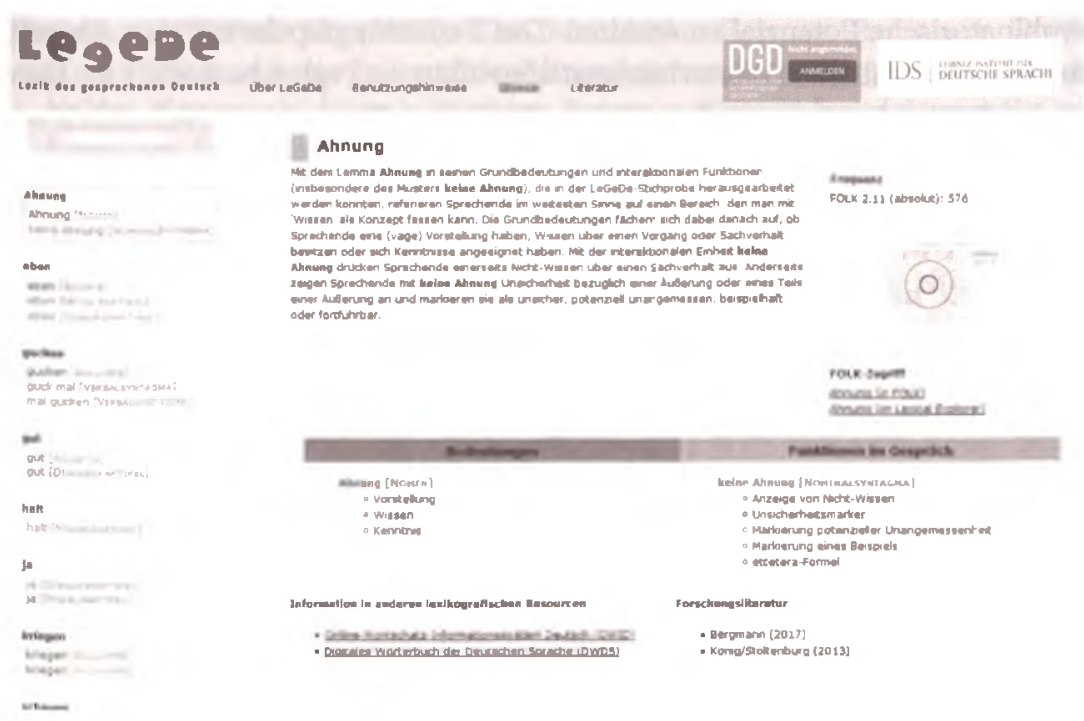

Abb. 13: Ausschnitt aus einem Überblicksartikel zu Ahnung aus der Beta-Version des LeGeDe-Prototyps

Anzeige von Nicht-Wissen

Mit kelne Ahnung zelgen Sprachende an, dass sie ken Wissen uber einen Sachverhatt butitzen (vol. dazu auch Ahnung un der Bedeutung Wirsanj.

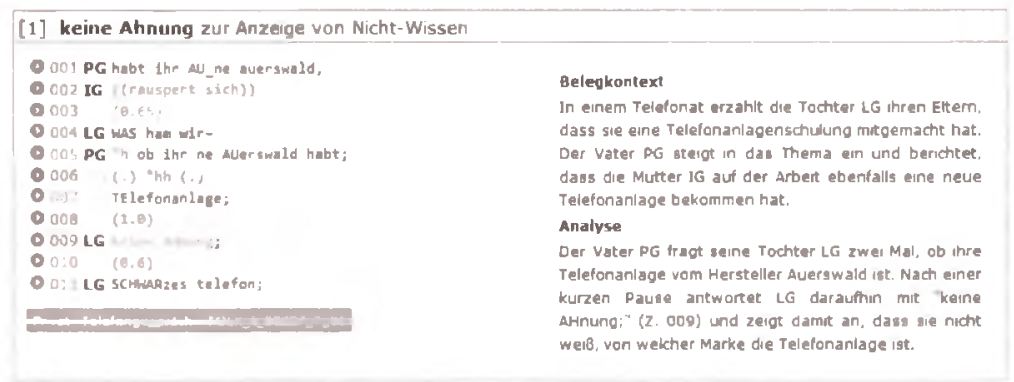

Abb. 14: Ausschnitt aus keine Ahnung in der Funktion, Anzeige von Nicht-Wissen aus der Beta-Version des LeGeDe-Prototyps

Wie in der Einleitung dieses Beitrags erläutert wurde, schlagen insbesondere LernerInnen des Deutschen als Fremdsprache in Wörterbüchern nach, um die Bedeutung eines Wortes zu entschlüsseln und Informationen über das 
kombinatorische Potenzial zu erhalten. Das Bedeutungsspektrum von Ahnung, das in herkömmlichen Wörterbüchern abgebildet ist (vgl. Abschnitt 3.1), kann bei der Betrachtung von authentischen Daten aus Textkorpora (schriftlich wie mündlich) in Teilen nachvollzogen werden. Die Analyse gesprochen-sprachlicher Daten aus Korpora zum gesprochenen Deutsch in der authentischen Interaktion wird in bislang vorhandenen Wörterbüchern allerdings eher vernachlässigt. Eine Hinzunahme auch von mündlicher (authentischer) Kommunikation (vgl. Abschnitt 3.2), wie es z.B. im LeGeDe-Projekt prototypisch gemacht wird, stellt eine Bereicherung in der Betrachtung des Bedeutungs- und Verwendungsspektrum eines Lexems dar. Eine Reflexion über Textsorten, in denen ein Lexem vorkommt, kann außerdem die Interpretation insgesamt, aber auch insbesondere von schriftsprachlichen Daten (vgl. Abschnitt 3.3) positiv beeinflussen: Im Ergebnis kann es zu einem Erkenntnisgewinn kommen, welche Verwendungsweisen in bestimmten Textsorten und bei einem Vergleich von geschriebenen und gesprochenen Daten für die jeweilige Sprachvarietät besonders typisch (oder auch angemessen) sind bzw, sein können.

\section{Literatur}

Bergmann, P. (2017): Gebrauchsprofile von weiß nich und keine Ahnung im Gespräch. Ein Blick auf nicht-responsive Vorkommen. In: Hardarik Blühdorn/ Arnulf Deppermann/Henrike Helmer/Thomas Spranz-Fogasy (Hgg.): Diskursmarker im Deutschen. Reflexionen und Analysen. Göttingen: Verlag für Gesprächsforschung. S. 157-182.

Eichinger, L. M. (2018): Keine Ahnung: Über etcetera-Formeln und Verwandtes, In: Laurent Gautier/ Pierre-Yves Modicom/Hélène Vinckel-Roisin (Hgg.): Diskursive Verfestigungen. Schnittstellen zwischen Morphosyntax, Phraseologie und Pragmatik im Deutschen und im Sprachvergleich. Festschrift für Martine Dalmas zum 65. Geburtstag. (= Konvergenz und Divergenz, Bd. 7). Berlin/Boston: de Gruyter. S. 243-262.

Feilke, H. (2009): Wörter und Wendungen: kennen, lernen, können. In: Praxis Deutsch 218. S. 4-14.

Koch, P./Oesterreicher, W. (2019): Mündlichkeit und Schriftlichkeit von Texten.

In: Nina Janich (Hg.): Textlinguistik. 15 Einführungen und eine Diskussion.

[2. aktualisierte und erweiterte Aufl.]. Tübingen: Narr Francke Attempto. S. 191-207. (= Narr Studienbücher).

König, K./Stoltenburg, B. (2013): „oder so", „und so“, „und so was", „und so weiter" etc. Eine interaktionale Perspektive auf Etcetera-Formeln. GIDI-Arbeitspapierreihe 48 (= Grammatik in der Interaktion). S. 1-35. 
Kupietz, M./Keibel, H. (2009): The Mannheim German Reference Corpus (DeReKo) as a basis for empirical linguistic research. In: Makoto Minegishi/Yuji Kawaguchi (Hgg.): Working Papers in Corpus-based Linguistics and Language Education, No. 3. Tokyo: Tokyo University of Foreign Studies (= TUFS). S. 53-59.

Kupietz, M./Belica, C./Keibel, H./Witt, A. (2010): The German Reference Corpus DeReKo: A primordial sample for linguistic research. In: Nicoletta Calzolari/Khalid Choukri/Bente Maegaard/ Joseph Mariani/Jan Odjik/Stelios Piperidis/Mike Rosner/Daniel Tapias (Hgg.): Proceedings of the 7th conference on International Language Resources and Evaluation (LREC 2010). Valletta, Malta: European Language Resources Association (= ELRA). S. 1848-1854.

Kupietz, M./Lüngen, H./Kamocki, P./Witt, A. (2018): The German Reference Corpus DeReKo: New Developments - New Opportunities. In: Nicoletta Calzolari/Khalid Choukri/Christopher Cieri/Thierry Declerck/Sara Goggi/Koiti Hasida/Hitoshi Isahara/Bente Maegaard/Joseph Mariani/Hélène Mazo/Asuncion Moreno/Jan Odijk/Stelios Piperidis/Takenobu Tokunaga (Hgg.): Proceedings of the Eleventh International Conference on Language Resources and Evaluation (LREC 2018). Miyazaki: European Language Resources Association (= ELRA), 2018. S. 4353-4360.

Meliss, M. (2015): Was suchen und finden Lerner des Deutschen als Fremdsprache in aktuellen Wörterbüchern? Auswertung einer Umfrage und Anforderungen an eine aktuelle DaF-Lernerlexikographie. In: Info DaF 42. S. 401-432.

Meliss, M./Möhrs, C. (2017): Die Entwicklung einer lexikografischen Ressource im Rahmen des Projektes LeGeDe. In: Sprachreport 4/2017. S. 42-52.

Meliss, M./Möhrs, C./Ribeiro Silveira, M. (2019): Anforderungen und Erwartungen an eine lexikografische Ressource des gesprochenen Deutsch aus der L2-Lernerperspektive. In: Lexicographica 34. S. 89-121.

Möhrs, C./Meliss, M./Batinić, D. (2017): LeGeDe - towards a corpus-based lexical resource of spoken German. In: Iztok Kosem/Carole Tiberius/Miloš Jakubíček/Jelena Kallas/Simon Krek/Vít Baisa (Hgg.): Electronic Lexicography in the 21st century. Proceedings of eLex 2017 Conference. 19-21 September 2017. Leiden, the Netherlands. Brno: Lexical Computing CZ s.r.o. S. 281-298.

Schlieben-Lange, B. (1983): Traditionen des Sprechens. Elemente einer pragmatischen Sprachgeschichtsschreibung. Stuttgart: Kohlhammer.

Schmidt, T. (2014a): Gesprächskorpora und Gesprächsdatenbanken am Beispiel von FOLK und DGD. In: Gesprächsforschung. Online-Zeitschrift zur verbalen Interaktion 15. S. 196-233.

Schmidt, T. (2014b): The Research and Teaching Corpus of Spoken German FOLK. In: Nicoletta Calzolari/Khalid Choukri/Thierry Declerck/Hrafn Loftsson/Bente Maegaard/Joseph Mariani/Asuncion Moreno/Jan Odijk/Stelios 
Piperidis (Hgg.): Proceedings of the 9th International Conference on Language Resources and Evaluation. 26-31 May 2014. Reykjavik, Iceland. Reykjavik: European Language Resources Association (= ELRA). S. 383-387.

Schmidt, T. (2017): DGD - Die Datenbank für Gesprochenes Deutsch. Mündliche Korpora am Institut für Deutsche Sprache (IDS) in Mannheim. In: Zeitschrift für Germanistische Linguistik 45. S. 451-463.

Taborek, J. (2019): Wörterbuchbenutzung von polnischen Germanistikstudierenden. In: Lexicographica 34. S. 207-219.

\section{Online-Quellen:}

DEREKo = Deutsches Referenzkorpus. URL: http://wwwl.ids-mannheim.de/kl/ projekte/korpora.html [letzter Zugriff: 02.08.2019].

COSMAS II: URL: https://www.ids-mannheim.de/cosmas2/ [letzter Zugriff: 02.08.2019].

DGD: URL: https://dgd.ids-mannheim.de/ [letzter Zugriff: 02.08.2019].

LeGeDe = Lexik des gesprochenen Deutsch. Projektwebseite: URL: http:// www.ids-mannheim.de/lexik/lexik-des-gesprochenen-deutsch.html [letzter Zugriff: 02.08.2019].

Lexical Explorer: URL: https://www.owid.de/lexex/ [letzter Zugriff: 02.08.2019].

OWID: URL: https://www.owid.de/ [letzter Zugriff: 02.08.2019].

OWIDplus: URL: https://www.owid.de/plus/ [letzter Zugriff: 02.08.2019].

\section{Wörterbuchquellen (gedruckt/online):}

Duden-DaF $=$ Duden Deutsch als Fremdsprache Standardwörterbuch (2010). 2., neu bearbeitete und erweiterte Auflage. Mannheim / Leipzig / Wien / Zürich: Dudenverlag.

Duden-online: URL: https://www.duden.de [letzter Zugriff: 02.08.2019]. LeGeDePrototyp: URL: https://www.owid.de/legede/ [letzter Zugriff: 12.02.2020].

LGW-DaF = Langenscheidt Großwörterbuch Deutsch als Fremdsprache (2015). München: Langenscheidt.

PONS-DaF = PONS Online Wörterbuch: URL: https://de.pons.com/ [letzter Zugriff: 02.08.2019]. 